\title{
Library Resources in the United States
}

\begin{abstract}
This is the third investigation of the distribution of American library resources, undertaken at approximately twenty-year intervals. The 1973 study reveals a phenomenal growth in library collections during the past eighteen years. During that period, the number of centers in the United States holding in excess of 500,000 volumes each increased from 109 to 265, and the total number of volumes in such centers expanded nearly threefold.
\end{abstract}

A of American library resources was reported in Louis Round Wilson's Geography of Reading. Based on sources published in 1935, Wilson discovered that there were in the United States at that time seventy-seven centers of not over fifty miles radius (airline) holding 500,000 volumes or more. ${ }^{1}$

The specifications stated by Wilson for computing his data were thus defined:

In general, the area included does not cover more than 50 miles (airline) from center. When a city could be attached to more than one center, the total number of volumes in the area and transportation facilities were considered in allocating it. Public or college libraries of less than 20,000 volumes and special libraries of less than 5,000 volumes were not included. State lines were not crossed except in special cases. Centers such as Newark were maintained separately. No city was chosen as a center unless it contained one library having at least 75,000 volumes. Preference was given to state capitals, or cities in which state universities were located. Metropolitan areas were selected unless the library center would fall elsewhere.

Robert B. Downs is Dean of Library Administration, Emeritus, University of Illinois, Urbana-Champaign.
Utilizing the same or similar sources of information, the Wilson study was updated about twenty years later. ${ }^{2}$ The second investigation revealed the relative ranks of the various centers, the number of volumes in each area in 1955 as compared to 1935 , and the increase in volumes. The number of centers was found to have grown from 77 to 109 during the twenty-year period.

In 1973, with assistance from a group of students in the University of Illinois Graduate School of Library Science, a second updating was undertaken. ${ }^{\circ}$ The results were little short of startling. As shown in Table 1, the number of centers in the United States holding in excess of 500,000 volumes each had jumped from 109 in 1955 to 265 in 1973. Even more striking, the total number of volumes in such centers had gone from $138,867,606$ volumes in 1935 to $289,355,391$ in 1955 to $724,045,043$ volumes in 1973-more than a fivefold increase in less than forty years. The growth rate during the past eighteen years is especially phenomenal.

Table 2 contains a breakdown of the 265 centers by states. Possessing the greatest number of centers with more than 500,000 volumes each was California, with a total of 21 , followed by Ohio with 18, New York and Texas with

- Under the chairmanship of Anne Billeter and Roland Streit. 
TABLE 1

Libraky Centers, of Not over 50 Mtles Radius (Amrline), Contanning 500,000 Volumes or More

\begin{tabular}{|c|c|c|c|c|c|c|}
\hline City & $\begin{array}{c}\text { Rank } \\
1973 \\
\end{array}$ & $\begin{array}{l}\text { Volumes in } \\
\text { Area, } 1973\end{array}$ & $\begin{array}{l}\text { Rank } \\
1955\end{array}$ & $\begin{array}{l}\text { Volumes in } \\
\text { Area, } 1955\end{array}$ & $\begin{array}{c}\text { Rank } \\
1935\end{array}$ & $\begin{array}{l}\text { Volumes in } \\
\text { Area, } 1935\end{array}$ \\
\hline New York City, N.Y. & 1 & $47,305,190$ & 2 & $24,688,777$ & 1 & $12,910,623$ \\
\hline Washington, D. C. & 2 & $39,728,774$ & $\hat{1}$ & $25,356,917$ & 2 & $11,744,966$ \\
\hline Boston, Mass. & $\overline{3}$ & $30,467,291$ & 3 & $18,334,377$ & $\overline{3}$ & $10,709,614$ \\
\hline Los Angeles, Calif. & 4 & $24,455,236$ & 5 & $10,096,635$ & 5 & $6,564,016$ \\
\hline Chicago, Ill. & 5 & $22,414,327$ & 4 & $12,519,393$ & 4 & $6,691,144$ \\
\hline Philadelphia, $\mathrm{Pa}$. & 6 & $14,759,903$ & 6 & $7,867,242$ & 6 & $4,805,252$ \\
\hline San Francisco, Calif. & 7 & $13,906,443$ & 7 & $6,901,607$ & 8 & $3,558,191$ \\
\hline Minneapolis, Minn. & 8 & $10,629,127$ & 11 & $4,988,889$ & 13 & $2,475,322$ \\
\hline San Jose; Stanford, Calif. & 9 & $10,350,412$ & 26 & $3,006,064$ & 22 & $1,551,791$ \\
\hline Newark, N.J. & 10 & $10,296,596$ & 9 & $6,024,375$ & 9 & $2,955,827$ \\
\hline Cleveland, Ohio & 11 & $9,142,394$ & 8 & $6,140,556$ & 7 & $3,835,889$ \\
\hline Pittsburgh, $\mathrm{Pa}$. & 12 & $9,076,306$ & 16 & $3,937,179$ & 16 & $2,136,108$ \\
\hline Detroit, Mich. & 13 & $8,322,385$ & 18 & $3,707,637$ & 25 & $1,404,736$ \\
\hline Baltimore, Md. & 14 & $8,257,188$ & 12 & $4,857,922$ & 12 & $2,477,779$ \\
\hline Dallas, Tex. (Ft. Worth, Denton) & 15 & $8,239,108$ & 37 & $1,963,176$ & 70 & 570,746 \\
\hline St. Louis, Mo. & 16 & $7,713,880$ & 22 & $3,217,632$ & 14 & $2,269,662$ \\
\hline New Haven, Conn. & 17 & $7,496,069$ & 10 & $5,330,221$ & 10 & $2,868,781$ \\
\hline Albany, N.Y. & 18 & $7,271,047$ & 13 & $4,498,344$ & 19 & $1,902,574$ \\
\hline \multicolumn{7}{|l|}{ Providence, R.I.: Fall River; New } \\
\hline Bedford, Mass. & 19 & $7,262,748$ & 19 & $3,646,088$ & 11 & $2,607,138$ \\
\hline Columbus, Ohio & 20 & $7,261,341$ & 14 & $4,119,799$ & 20 & $1,813,637$ \\
\hline Buffalo, N.Y. & 21 & $6,504,369$ & 29 & $2,809,211$ & 27 & $1,341,455$ \\
\hline Springfield, Mass. & 22 & $5,729,951$ & 23 & $3,170,131$ & 17 & $2,099,229$ \\
\hline Cincinnati, Ohio & 23 & $5,592,171$ & 17 & $3,750,316$ & 18 & $2,066,825$ \\
\hline Seattle, Wash. & 24 & $5,525,875$ & 24 & $3,122,956$ & 36 & $1,064,818$ \\
\hline San Diego, Calif. & 25 & $5,277,346$ & 70 & $1,010,631$ & 77 & 500,383 \\
\hline Urbana, Ill. & 26 & $5,199,575$ & 25 & $3,072,034$ & 33 & $1,184,928$ \\
\hline Ann Arbor, Mich. & 27 & $5,103,299$ & 28 & $2,884,529$ & 35 & $1,074,274$ \\
\hline Milwaukee, Wis. & 28 & $5,074,643$ & 31 & $2,673,066$ & 21 & $1,565,732$ \\
\hline Kansas City, Mo. and Kans. & 29 & $5,021,465$ & 40 & $1,937,367$ & 47 & 788,837 \\
\hline Chapel Hill; Durham, N.C. & 30 & $4,996,743$ & 21 & $3,337,247$ & 34 & $1,091,858$ \\
\hline Atlanta, $\mathrm{Ga}$. & 31 & $4,873,274$ & 42 & $1,865,435$ & 54 & 715,842 \\
\hline Sacramento, Calif. & 32 & $4,857,605$ & 38 & $1,958,441$ & 23 & $1,534,107$ \\
\hline Madi & 33 & $4,813,740$ & 36 & $2,155,846$ & 26 & $1,341,899$ \\
\hline Evanston, III. & 34 & $4,683,075$ & & & & \\
\hline Ithaca, N.Y. & 35 & $4,654,116$ & 32 & $2,573,378$ & 31 & $1,264,920$ \\
\hline Nashville, Tenn. & 36 & $4,600,596$ & 51 & $1,526,868$ & 46 & 791,242 \\
\hline Houston, Tex. & 37 & $4,596,864$ & 63 & $1,231,685$ & & \\
\hline New Orleans, La. & 38 & $4,301,324$ & 49 & $1,625,299$ & 56 & 702 \\
\hline Rochester, N.Y. & 39 & $4,279,592$ & 46 & $1,836,532$ & 24 & $1,515,438$ \\
\hline Belts & 40 & $4,266,040$ & & & & \\
\hline Hartford, Conn. & 41 & $4,251,603$ & 20 & 38 & 58 & 217 \\
\hline Aus & 42 & 4,21 & 41 & & 49 & 391 \\
\hline Colo. & 43 & $4,150,133$ & 30 & $2,715,259$ & 32 & $1,212,159$ \\
\hline Mich. & 44 & $3,918,201$ & 34 & $2,351,614$ & 45 & 797,736 \\
\hline Worcest & 45 & $3,809,191$ & 35 & $2,341,469$ & 28 & $1,315,636$ \\
\hline Salt Lake City, Utah & 46 & $3,773,746$ & 65 & $1,218,566$ & & \\
\hline Orange, Calif. & 47 & $3,564,404$ & & & & \\
\hline New Brunswick, N.J. & 48 & $3,476,954$ & & & & \\
\hline Miami, Fla. & 49 & $3,421,391$ & 88 & 757,906 & & \\
\hline Princet & 50 & $3,367,802$ & 15 & $4,040,143$ & 15 & $2,150,512$ \\
\hline olis, Ind. & 51 & $3,326,594$ & 27 & $3,000,499$ & 30 & $1,266,031$ \\
\hline Oklahoma City, Okla. & 52 & $3,242,373$ & 50 & $1,550,821$ & 68 & 604.140 \\
\hline Dayton, Ohio & 53 & $3,189,778$ & 48 & $1,637,991$ & 41 & 955,198 \\
\hline Phoenix, Ariz. & 54 & $3,171,641$ & 84 & 815,778 & & \\
\hline Syracuse, N.Y. & 55 & $3,039,009$ & 77 & 906,084 & 57 & 695,565 \\
\hline Bloomington, Ind. & 56 & $2,932,410$ & & & & \\
\hline Bridgeport, Conn. & 57 & $2,917,130$ & 61 & $1,240,975$ & 65 & 607,834 \\
\hline & & & & & & \\
\hline
\end{tabular}


TABLE 1-Continued

\begin{tabular}{|c|c|c|c|c|c|c|}
\hline City & $\begin{array}{l}\text { Rank } \\
1973\end{array}$ & $\begin{array}{l}\text { Volumes in } \\
\text { Area, } 1973\end{array}$ & $\begin{array}{l}\text { Rank } \\
1955\end{array}$ & $\begin{array}{l}\text { Volumes in } \\
\text { Area, } 1955\end{array}$ & $\begin{array}{l}\text { Rank } \\
1935\end{array}$ & $\begin{array}{l}\text { Volumes in } \\
\text { Area, } 1935\end{array}$ \\
\hline Knoxville, Tenn. & 59 & $2,821,730$ & 85 & 801,299 & & \\
\hline Richmond, Va. & 60 & $2,777,141$ & 53 & $1,441,119$ & 61 & 651,842 \\
\hline Portland, Oreg. & 61 & $2,776,170$ & 59 & $1,322,670$ & 53 & 759,320 \\
\hline Norfolk, Va. & 62 & $2,762,843$ & 104 & 590,478 & & \\
\hline Honolulu, Hawaii & 63 & $2,706,530$ & & & & \\
\hline $\begin{array}{l}\text { Springfield, Ill. } \\
\text { Tucson, Ariz. }\end{array}$ & $\begin{array}{l}64 \\
65\end{array}$ & $\begin{array}{l}2,659,113 \\
2,542,726\end{array}$ & 44 & $1,851,364$ & 60 & 667,247 \\
\hline Toledo, Ohio & 66 & $2,460,024$ & 82 & 818,293 & 63 & 620,711 \\
\hline Columbia, Mo. & 67 & $2,449,138$ & 55 & $1,413,600$ & 55 & 715,829 \\
\hline Columbia, S.C. & 68 & $2,442,470$ & 73 & 960,247 & & \\
\hline Charlottesville, Va. & 69 & $2,359,824$ & 67 & $1,069,935$ & & \\
\hline Poughkeepsie, N.Y. & 70 & $2,346,182$ & 81 & 822,856 & 64 & 613,535 \\
\hline San Antonio, Tex. & $\begin{array}{l}71 \\
72\end{array}$ & $2,311,777$ & 87 & 782,248 & & \\
\hline Bethlehem, $\mathrm{Pa}$. & 73 & $2,276,772$ & 68 & $1,048,181$ & 50 & 783,274 \\
\hline Louisville, Ky. & 74 & $2,275,897$ & 71 & $1,003,900$ & 71 & 537,494 \\
\hline Santa Barbara, Calif. & 75 & $2,238,939$ & 101 & 623,121 & & \\
\hline South Bend, Ind. & 76 & $2,221,157$ & 76 & 991,314 & & \\
\hline State College, Pa. & 77 & $2,202,977$ & 92 & 715,896 & & \\
\hline Harrisburg, Pa. & 78 & $2,195,208$ & 47 & $1,743,178$ & 43 & 902,802 \\
\hline Charlotte, N.C. & 79 & $2,153,951$ & 96 & 670,739 & & \\
\hline Claremont, Calif. & 80 & $2,129,149$ & & & & \\
\hline Bethesda, Md. & 81 & $2,128,960$ & & & & \\
\hline Baton Rouge, La. & 82 & $2,102,149$ & 54 & $1,415,255$ & & \\
\hline Gainesville, Fla. & 83 & $2,102,081$ & 94 & 686,243 & & \\
\hline Memphis, Tenn. & 84 & $2,087,296$ & & & & \\
\hline Ft. Wayne, Ind. & 85 & $2,049,834$ & 66 & $1,133,926$ & & \\
\hline Des Moines, Iowa & 86 & $2,033,167$ & 43 & $1,851,960$ & 40 & 972,814 \\
\hline Lowell, Mass. & 87 & $2,020,728$ & 105 & 580,119 & 48 & 784,843 \\
\hline Lincoln, Nebr. & 88 & $2,003,156$ & 62 & $1,237,610$ & 37 & $1,031,052$ \\
\hline Roanoke, Va. & 89 & $1,966,640$ & 79 & 859,511 & & \\
\hline Fresno, Calif. & 90 & $1,943,257$ & 72 & 971,751 & 38 & 995,404 \\
\hline Gary, Ind. & 91 & $1,897,864$ & 89 & 739,501 & & \\
\hline Greenville, S.C. & 92 & $1,862,782$ & 98 & 658,138 & & \\
\hline Iowa City, Iowa & 93 & $1,823,655$ & 56 & $1,401,880$ & 59 & 679,405 \\
\hline Wilmington, Del. & 94 & $1,807,400$ & 100 & 629,775 & & \\
\hline Birmingham, Ala. & 95 & $1,798,117$ & 57 & $1,374,977$ & 76 & 508,381 \\
\hline Charleston, W. Va. & 96 & $1,788,628$ & 95 & 678,681 & & \\
\hline Morgantown, W. Va. & 97 & $1,745,301$ & 102 & 596,929 & & \\
\hline Lafayette, Ind. & 98 & $1,741,722$ & 69 & $1,020,870$ & 75 & 513,855 \\
\hline Athens, Ga. & 99 & $1,736,052$ & & & & \\
\hline Utica, N.Y. & 100 & $1,733,360$ & 75 & 917,844 & 74 & 514,373 \\
\hline Greensboro, N.C. & 101 & $1,729,099$ & & & & \\
\hline Grand Rapids, Mich. & 102 & $1,713,913$ & 60 & $1,299,376$ & 52 & 775,680 \\
\hline Boulder, Colo. & 103 & $1,713,287$ & & & & \\
\hline Lexington, Ky. & 104 & $1,711,201$ & 52 & $1,475,022$ & 69 & 578,806 \\
\hline Lawrence, Kans. & 105 & $1,703,603$ & & & & \\
\hline Carlisle Barracks, $\mathrm{Pa}$. & 106 & $1,683,676$ & & & & \\
\hline San Bernardino, Calif. & 107 & $1,677,737$ & & & & \\
\hline Kalamazoo, Mich. & 108 & $1,673,680$ & & & & \\
\hline Carbondale, Ill. & 109 & $1,672,492$ & & & & \\
\hline Muncie, Ind. & 110 & $1,662,381$ & & & & \\
\hline Topeka, Kans. & 111 & $1,660,161$ & 33 & $2,505,793$ & 29 & $1,293,901$ \\
\hline Mount Pleasant, Mich. & 112 & $1,637,231$ & & & & \\
\hline Bowling Green, Ohio & 113 & $1,636,219$ & & & & \\
\hline Winston-Salem, N.C. & 114 & $1,618,747$ & & & & \\
\hline Omaha, Nebr. & 115 & $1,616,670$ & 91 & 724,850 & & \\
\hline Akron, Ohio & 116 & $1,595,648$ & 39 & $1,937,456$ & 44 & 799,509 \\
\hline Rockville, Md. & 117 & $1,594,171$ & & & & \\
\hline Tallahassee, Fla. & 118 & $1,566,269$ & 109 & 537,755 & & \\
\hline Stockton, Calif. & 119 & $1,557,424$ & & & & \\
\hline Little Rock, Ark. & 120 & $1,547,068$ & 90 & 736,870 & & \\
\hline
\end{tabular}


TABLE 1-Continued

\begin{tabular}{|c|c|c|c|c|c|c|}
\hline City & $\begin{array}{l}\text { Rank } \\
1973\end{array}$ & $\begin{array}{l}\text { Volumes in } \\
\text { Area, } 1973\end{array}$ & $\begin{array}{l}\text { Rank } \\
1955\end{array}$ & $\begin{array}{l}\text { Volumes in } \\
\text { Area, } 1955\end{array}$ & $\begin{array}{l}\text { Rank } \\
1935\end{array}$ & $\begin{array}{l}\text { Volumes in } \\
\text { Area, } 1935\end{array}$ \\
\hline Lancaster, $\mathrm{Pa}$. & 121 & $1,526,941$ & & & & \\
\hline Albuquerque, N. Mex. & 122 & $1,518,453$ & & & 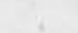 & \\
\hline Canton, Ohio & 123 & $1,512,423$ & & & & \\
\hline Tulsa, Okla. & 124 & $1,508,558$ & 93 & 693,836 & & \\
\hline De Kalb, Ill. & 125 & $1,477,499$ & & & & \\
\hline Stillwater, Okla. & 126 & $1,461,912$ & & & & \\
\hline Riverside, Calif. & 127 & $1,444,869$ & & & & \\
\hline Oberlin, Ohio & 128 & $1,411,600$ & & & & \\
\hline Orlando, Fla. & 129 & $1,404,908$ & & & & \\
\hline Raleigh, N.C. & 130 & $1,403,434$ & & & & \\
\hline Jackson, Miss. & 131 & $1,395,780$ & & & & \\
\hline Ft. Collins, Colo. & 132 & $1,389,566$ & & & & \\
\hline Youngstown, Ohio & 133 & $1,382,065$ & & & & \\
\hline Wichita, Kans. & 134 & $1,372,265$ & & & & \\
\hline Lubbock, Tex. & 135 & $1,365,848$ & & & & \\
\hline Wilkes-Barre, $\mathrm{Pa}$. & 136 & $1,363,365$ & 108 & 546,564 & & \\
\hline Oshkosh, Wis. & 137 & $1,355,814$ & 78 & 892,690 & & \\
\hline Augusta, Maine & 138 & $1,341,908$ & 64 & $1,230,769$ & 39 & 989,944 \\
\hline Portland, Maine & 139 & $1,335,429$ & & & & \\
\hline Hanover, N.H. & 140 & $1,324,377$ & 80 & 831,089 & 73 & 523,641 \\
\hline Schenectady, N.Y. & 141 & $1,314,360$ & & & & \\
\hline Eugene, Oreg. & 142 & $1,305,048$ & & & & \\
\hline Trenton, N.J. & 143 & $1,296,306$ & & & & \\
\hline Tuscaloosa, Ala. & 144 & $1,287,680$ & & & & \\
\hline Terre Haute, Ind. & 145 & $1,255,784$ & & & & \\
\hline Tacoma, Wash. & 146 & $1,253,235$ & & & & \\
\hline Hayward, Calif. & 147 & $1,246,153$ & & & & \\
\hline Erie, $\mathrm{Pa}$. & 148 & $1,209,573$ & 107 & 547,168 & & \\
\hline Springfield, Ohio & 149 & $1,195,714$ & & & & \\
\hline Colorado Springs, Colo. & 150 & $1,193,829$ & & & & \\
\hline Concord, N.H. & 151 & $1,185,437$ & 58 & $1,344,355$ & 51 & 782,818 \\
\hline Storrs, Conn. & 152 & $1,173,821$ & & & & \\
\hline Davis, Calif. & 153 & $1,169,537$ & & & & \\
\hline Annapolis, Md. & 154 & $1,166,092$ & & & & \\
\hline Tampa, Fla. & 155 & $1,161,051$ & & & & \\
\hline Spokane, Wash. & 156 & $1,151,320$ & & & & \\
\hline Athens, Ohio & 157 & $1,144,112$ & 97 & 664,610 & & \\
\hline Fort Monmouth, N.J. & 158 & $1,125,176$ & & & & \\
\hline Montgomery, Ala. & 159 & $1,110,148$ & 86 & 797,701 & & \\
\hline Kent, Ohio & 160 & $1,100,213$ & & & & \\
\hline Provo, Utah & 161 & $1,095,405$ & & & & \\
\hline Monterey, Calif. & 162 & $1,093,615$ & & & & \\
\hline Auburn, Ala. & 163 & $1,086,535$ & & & & \\
\hline Olympia, Wash. & 164 & $1,069,806$ & & & & \\
\hline Oxford, Ohio & 165 & $1,067,620$ & & & & \\
\hline Salem, Oreg. & 166 & $1,043,685$ & & & & \\
\hline Jacksonville, Fla. & 167 & $1,043,047$ & & i & & \\
\hline Ames, Iowa & 168 & $1,039,313$ & & & & \\
\hline Burlington, Vt. & 169 & $1,037,185$ & & & & \\
\hline Greenville, N.C. & 170 & $1,023,298$ & & & & \\
\hline Ruston, La. & 171 & $1,021,471$ & & & & \\
\hline Binghamton, N.Y. & 172 & $1,007,284$ & & & & \\
\hline Waco, Tex. & $17 \overline{3}$ & 997,516 & & & & \\
\hline Evansville, Ind. & 174 & 990,301 & 106 & 560,300 & & \\
\hline Bangor, Maine & 175 & 986,053 & & & & \\
\hline Springfield, Mo. & 176 & 979,513 & & & & \\
\hline Fayetteville, Ark. & 177 & 974,319 & & & & \\
\hline El Paso, Tex. & 178 & 956,189 & & & & \\
\hline St. Petersburg, Fla. & 179 & 955,628 & & & & \\
\hline Reno, Nev. & 180 & 942,792 & & & & \\
\hline Middletown, Conn. & 181 & 939,449 & & & & \\
\hline Wooster, Ohio & 182 & 918,807 & & & & \\
\hline
\end{tabular}


TABLE 1-Continued

\begin{tabular}{|c|c|c|c|c|c|c|}
\hline City & $\begin{array}{l}\text { Rank } \\
1973\end{array}$ & $\begin{array}{l}\text { Volumes in } \\
\text { Area, } 1973\end{array}$ & $\begin{array}{l}\text { Rank } \\
1955\end{array}$ & $\begin{array}{l}\text { Volumes in } \\
\text { Area, } 1955\end{array}$ & $\begin{array}{l}\text { Rank } \\
1935\end{array}$ & $\begin{array}{l}\text { Volumes in } \\
\text { Area, } 1935\end{array}$ \\
\hline $\begin{array}{l}\text { Chattanooga, Tenn. } \\
\text { Montpelier, Vt. } \\
\text { Canyon, Tex. } \\
\text { Camden, N.J. } \\
\text { Normal, Ill. } \\
\text { Green Bay, Wis. } \\
\text { Pullman, Wash. } \\
\text { Macomb, Ill. } \\
\text { Delaware, Ohio } \\
\text { Peoria, Ill. } \\
\text { Pleasant Hill, Calif. } \\
\text { Cedar Falls, Iowa } \\
\text { Huntsville, Ala. } \\
\text { Boise, Idaho } \\
\text { Chico, Calif. } \\
\text { Moscow, Idaho } \\
\text { Columbus, Ga. } \\
\text { College Station, Tex. } \\
\text { Mankato, Minn. } \\
\text { Macon, Ga. } \\
\text { St. Cloud, Minn. } \\
\text { Lima, Ohio } \\
\text { Santa Fe, N. Mex. } \\
\text { Durham, N.H. } \\
\text { Edwardsville, Ill. } \\
\text { Jefferson City, Mo. } \\
\text { Laramie, Wyo. } \\
\text { Asheville, N.C. } \\
\text { Missoula, Mont. } \\
\text { Corpus Christi, Tex. } \\
\text { Santa Cruz, Calif. } \\
\text { Manhattan, Kans. } \\
\text { Corvallis, Oreg. } \\
\text { Elmira, N.Y. } \\
\text { Charleston, S.C. } \\
\text { Winona, Minn. } \\
\text { Walla Walla, Wash. } \\
\text { Gadsden, Ala. } \\
\text { Bakersfield, Calif. } \\
\text { Albany, Ga. } \\
\text { Stevens Point, Wis. } \\
\text { Potsdam, N.Y. } \\
\text { Savannah, Ga. } \\
\text { Zanesville, Ohio } \\
\text { University, Miss. } \\
\text { Pensacola, Fla. } \\
\text { Dubuque, Iowa } \\
\text { Abilene, Tex. } \\
\text { Cedar Rapids, Iowa } \\
\text { Richmond, Ky. } \\
\text { Galveston, Tex. } \\
\text { Davenport, Iowa } \\
\text { Johnson City, Tenn. } \\
\text { New London, Conn. } \\
\text { Fayetteville, N.C. } \\
\text { Manchester, N.H. } \\
\text { Hattiesburg, Miss. } \\
\text { San Luis Obispo, Calif. } \\
\text { Augusta, Ga. } \\
\text { Commerce, Tex. } \\
\text { Pocatello, Idaho } \\
\text { Mobile, Ala. } \\
\text { ola }\end{array}$ & $\begin{array}{l}183 \\
184 \\
185 \\
186 \\
187 \\
188 \\
189 \\
190 \\
191 \\
192 \\
193 \\
194 \\
195 \\
196 \\
197 \\
198 \\
199 \\
200 \\
201 \\
202 \\
203 \\
204 \\
205 \\
206 \\
207 \\
208 \\
209 \\
210 \\
211 \\
212 \\
213 \\
214 \\
215 \\
216 \\
217 \\
218 \\
219 \\
220 \\
221 \\
222 \\
223 \\
224 \\
225 \\
226 \\
227 \\
228 \\
229 \\
230 \\
231 \\
232 \\
233 \\
234 \\
235 \\
236 \\
237 \\
238 \\
239 \\
240 \\
241 \\
242 \\
243 \\
244\end{array}$ & $\begin{array}{l}914,241 \\
913,346 \\
899,747 \\
898,206 \\
891,302 \\
886,991 \\
853,458 \\
847,790 \\
845,605 \\
842,319 \\
839,847 \\
834,364 \\
831,572 \\
830,300 \\
829,788 \\
822,006 \\
813,333 \\
812,595 \\
812,032 \\
807,907 \\
795,881 \\
788,486 \\
788,186 \\
788,142 \\
785,830 \\
775,898 \\
774,593 \\
766,151 \\
763,420 \\
747,629 \\
747,168 \\
742,916 \\
741,003 \\
740,676 \\
733,163 \\
725,233 \\
714,114 \\
713,584 \\
710,623 \\
703,748 \\
699,444 \\
698,432 \\
697,804 \\
697,733 \\
690,916 \\
685,479 \\
681,616 \\
672,570 \\
667,926 \\
662,418 \\
661,734 \\
657,329 \\
656,722 \\
647,307 \\
634,126 \\
627,143 \\
625,371 \\
615,178 \\
611,611 \\
605,408 \\
601,187 \\
574,403\end{array}$ & 103 & 817,470 & 42 & $\begin{array}{l}903,154 \\
641,660\end{array}$ \\
\hline
\end{tabular}


TABLE 1 - Continued

\begin{tabular}{|c|c|c|c|c|c|c|}
\hline City & $\begin{array}{l}\text { Rank } \\
1973\end{array}$ & $\begin{array}{l}\text { Volumes in } \\
\text { Area, } 1973\end{array}$ & $\begin{array}{l}\text { Rank } \\
1955\end{array}$ & $\begin{array}{l}\text { Volumes in } \\
\text { Area, } 1955\end{array}$ & $\begin{array}{l}\text { Rank } \\
1935\end{array}$ & $\begin{array}{l}\text { Volumes in } \\
\text { Area, } 1935\end{array}$ \\
\hline Sioux City, Iowa & 245 & 562,026 & & & & \\
\hline Logan, Utah & 246 & 561,698 & & & & \\
\hline Bozeman, Mont. & 247 & 561,331 & & & & \\
\hline Warrensburg, Mo. & 248 & 560,133 & & & & \\
\hline Frankfort, Ky. & 249 & 557,694 & & & & \\
\hline Rock Island, IIl. & 250 & 556,179 & 99 & 654,067 & & \\
\hline Alfred, N.Y. & 251 & 542,819 & & & & \\
\hline State University, Ark. & 252 & 541,639 & & & & \\
\hline Boone, N.C. & 253 & 538,170 & & & & \\
\hline LaFayette, La. & 254 & 537,511 & & & & \\
\hline Eureka, Calif. & 255 & 537,197 & & & & \\
\hline State College, Miss. & 256 & 534,827 & & & & \\
\hline Martin, Tenn. & 257 & 528,921 & & & & \\
\hline Vermillion, S. Dak. & 258 & 523,096 & & & & \\
\hline Helena, Mont. & 259 & 522,182 & & & & \\
\hline Hanover, Ind. & 260 & 522,027 & & & & \\
\hline Huntsville, Tex. & 261 & 521,186 & & & & \\
\hline Fairfield, Iowa & 262 & 516,266 & & & & \\
\hline Grand Forks, N. Dak. & 263 & 509,519 & & & & \\
\hline Emporia, Kans. & 264 & 502,946 & & & & \\
\hline Medford, Oreg. & 265 & 500,723 & & & & \\
\hline TOTAL & & $724,045,043$ & & $289,355,391$ & & $138,867,606$ \\
\hline
\end{tabular}

(The apparent loss of volumes in some centers is the result of the establishment of new centers and subtraction of their holdings from older centers.)

TABLE 2

Library Centers Arranged by States

\begin{tabular}{|c|c|c|c|}
\hline Alabama (7) & & Sacramento & $4,857,605$ \\
\hline Auburn & $1,086,535$ & San Bernardino & $1,677,737$ \\
\hline Birmingham & $1,798,117$ & San Diego & $5,277,346$ \\
\hline Gadsden & 713,584 & San Francisco & $13,906,443$ \\
\hline Huntsville & 831,572 & San Jose-Stanford & $10,350,412$ \\
\hline Mobile & 574,403 & San Luis Obispo & 615,178 \\
\hline Montgomery & $1,110,148$ & Santa Barbara & $2,238,939$ \\
\hline Tuscaloosa & $1,287,680$ & Santa Cruz & 747,168 \\
\hline Alaska (0) & & Stockton & $1,557,424$ \\
\hline Arizona (2) & & Colorado (4) & \\
\hline Phoenix & $3,171,641$ & Boulder & $1,713,287$ \\
\hline Tucson & $2,542,726$ & Colorado Springs & $1,193,829$ \\
\hline Arkansas (3) & & Denver & $4,150,133$ \\
\hline Fayetteville & 974,319 & Ft. Collins & $1,389,566$ \\
\hline Little Rock & $1,547,068$ & Connecticut (6) & \\
\hline State University & 541,639 & Bridgeport & $2,917,130$ \\
\hline California (21) & & Hartford & $4,251,603$ \\
\hline Bakersfield & 710,623 & Middletown & 939,449 \\
\hline Chico & 829,788 & New Haven & $7,496,069$ \\
\hline Claremont & $2,129,149$ & New London & 647,307 \\
\hline Davis & $1,169,537$ & Storrs & $1,173,821$ \\
\hline Eureka & 537,197 & Delaware (1) & \\
\hline Fresno & $1,943,257$ & Wilmington & $1,807,400$ \\
\hline Hayward & $1,246,153$ & District of Columbia (1) & \\
\hline Los Angeles & $24,455,236$ & D.C. & $39,728,774$ \\
\hline Monterey & $1,093,615$ & Florida (8) & \\
\hline Orange & $3,564,404$ & Gainesville & $2,102,081$ \\
\hline Pleasant Hill & 839,847 & Jacksonville & $1,043,047$ \\
\hline Riverside & $1,444,869$ & Miami & $3,553,788$ \\
\hline
\end{tabular}


TABLE 2-Continued

\begin{tabular}{|c|c|c|c|}
\hline $\begin{array}{l}\text { Orlando } \\
\text { Pensacola } \\
\text { St. Petersburg }\end{array}$ & $\begin{array}{r}1,404,908 \\
685,479 \\
955,628\end{array}$ & $\begin{array}{l}\text { LaFayette } \\
\text { New Orleans } \\
\text { Ruston }\end{array}$ & $\begin{array}{r}537,511 \\
4,301,324 \\
1,021,471\end{array}$ \\
\hline Tallahassee & $1,566,269$ & Maine (3) & \\
\hline Tampa & $1,161,051$ & Augusta & $1,341,908$ \\
\hline Georgia (7) & & Bangor, & 986,053 \\
\hline $\begin{array}{l}\text { Albany } \\
\text { Athens }\end{array}$ & 703,748 & Portland & $1,335,429$ \\
\hline $\begin{array}{l}\text { Athens } \\
\text { Atlanta }\end{array}$ & $\begin{array}{l}1,736,052 \\
4,873,274\end{array}$ & $\begin{array}{c}\text { Maryland (5) } \\
\text { Annapolis }\end{array}$ & $1,166,092$ \\
\hline Augusta & 611,611 & Baltimore & $8,257,188$ \\
\hline Columbus & 813,333 & Beltsville & $4,266,040$ \\
\hline Macon & 807,907 & Bethesda & $2,128,960$ \\
\hline Savannah & 697,804 & Rockville & $1,594,171$ \\
\hline Hawaii (1) & & Massachusetts (4) & \\
\hline Honolulu & $2,706,530$ & Boston & $30,467,291$ \\
\hline Idaho (3) & & Lowell & $2,020,728$ \\
\hline $\begin{array}{l}\text { Boise } \\
\text { Moscow }\end{array}$ & 830,300 & $\begin{array}{l}\text { Springfield } \\
\text { Worcester }\end{array}$ & $\begin{array}{l}5,729,951 \\
3,809,191\end{array}$ \\
\hline $\begin{array}{l}\text { Moscow } \\
\text { Pocatello }\end{array}$ & $\begin{array}{l}822,000 \\
601,187\end{array}$ & $\begin{array}{l}\text { Worcester } \\
\text { Michigan (6) }\end{array}$ & $3,009,191$ \\
\hline Illinois (11) & & Ann Arbor & $5,103,299$ \\
\hline Carbondale & $1,672,492$ & Detroit & $8,322,385$ \\
\hline Chicago & $22,414,327$ & Grand Rapids & $1,713,913$ \\
\hline De Kalb & $1,477,499$ & Kalamazoo & $1,673,680$ \\
\hline Evanston & $4,683,075$ & Lansing & $3,918,201$ \\
\hline Edwardsville & 785,830 & Mount Pleasant & $1,637,231$ \\
\hline Macomb & 847,790 & Minnesota (4) & \\
\hline Normal & 891,302 & Minneapolis & $10,629,127$ \\
\hline Peoria & 842,319 & Mankato & 812,032 \\
\hline Rock Island & 556,179 & St. Cloud & 795,881 \\
\hline Springfield & $2,659,113$ & Winona & 725,233 \\
\hline Urbana & $5,199,575$ & Mississippi (4) & \\
\hline Indiana (10) & & Hattiesburg & 625,371 \\
\hline Bloomington & $2,932,410$ & Jackson & $1,395,780$ \\
\hline Evansville & 990,301 & State College & 534,827 \\
\hline Ft. Wayne & $2,049,834$ & University & 690,916 \\
\hline Gary & $1,897,864$ & Missouri (6) & \\
\hline Hanover & 522,027 & Columbia & $2,449,138$ \\
\hline Indianapolis & $3,326,594$ & Jefferson City & 775,898 \\
\hline Lafayette & $1,741,722$ & Kansas City (Mo. \& Kans.) & $5,021,465$ \\
\hline Muncie & $1,662,381$ & St. Louis & $7,713,880$ \\
\hline South Bend & $2,221,157$ & Springfield & 979,513 \\
\hline Terre Haute & $1,255,784$ & Warrensburg & 560,133 \\
\hline Iowa $(9)$ & & Montana (3) & \\
\hline Ames & $1,039,313$ & Bozeman & 561,331 \\
\hline Cedar Falls & 834,364 & Helena & 522,182 \\
\hline Cedar Rapids & 667,926 & Missoula & 763,420 \\
\hline Davenport & 657,329 & Nebraska (2) & \\
\hline Des Moines & $2,033,167$ & Lincoln & $2,003,156$ \\
\hline Dubuque & 681,616 & Omaha & $1,616,670$ \\
\hline Fairfield & 516,266 & Nevada (1) & \\
\hline Iowa City & $1,823,655$ & Reno & 942,792 \\
\hline Sioux City & 562,026 & New Hampshire (4) & \\
\hline Kansas (5) & & Concord & $1,185,437$ \\
\hline Emporia & 502,946 & Durham & 788,142 \\
\hline Lawrence & $1,703,603$ & Hanover & $1,324,377$ \\
\hline Manhattan & 742,916 & Manchester & 627,143 \\
\hline Topeka & $1,660,161$ & New Jersey (6) & \\
\hline Wichita & $1,372,265$ & Camden & 898,206 \\
\hline Kentucky (4) & & Fort Monmouth & $1,125,176$ \\
\hline Frankfort & 557,694 & New Brunswick & $3,476,954$ \\
\hline Lexington & $1,711,201$ & Newark & $10,296,596$ \\
\hline Louisville & $2,275,897$ & Princeton & $3,367,802$ \\
\hline Richmond & 662,418 & Trenton & $1,296,306$ \\
\hline Louisiana (4) & & New Mexico (2) & \\
\hline Baton Rouge & $2,102,149$ & Albuquerque & $1,518,453$ \\
\hline
\end{tabular}


TABLE 2-Continued

\begin{tabular}{|c|c|c|c|}
\hline Santa $\mathrm{Fe}$ & 788,186 & Pittsburgh & $9,076,306$ \\
\hline New York (14) & & State College & $2,202,977$ \\
\hline Albany & $7,271,047$ & Wilkes-Barre & $1,363,365$ \\
\hline Alfred & 542,819 & Rhode Island (1) & \\
\hline $\begin{array}{l}\text { Binghamton } \\
\text { Buffalo }\end{array}$ & $\begin{array}{l}1,007,284 \\
6,504,369\end{array}$ & $\begin{array}{l}\text { Providence } \\
\text { South Carolina (3) }\end{array}$ & $7,262,748$ \\
\hline Elmira & $\begin{array}{r}0,004,309 \\
740,676\end{array}$ & $\begin{array}{l}\text { Charleston } \\
\text { Cout }\end{array}$ & 733,163 \\
\hline Ithaca & $4,654,116$ & Columbia & $2,442,470$ \\
\hline New York City & $47,305,190$ & Greenville & $1,862,782$ \\
\hline Potsdam & 698,432 & South Dakota (1) & \\
\hline Poughkeepsie & $2,346,182$ & Vermillion & 523,096 \\
\hline Rochester & $4,279,592$ & Tennessee (6) & \\
\hline Schenectady & $1,314,360$ & Chattanooga & 914,241 \\
\hline Stony Brook & $2,283,336$ & Johnson City & 656,722 \\
\hline Syracuse & $3,039,009$ & Knoxville & $2,821,730$ \\
\hline Útica & $1,733,360$ & Martin & 528,921 \\
\hline North Carolina (9) & & Memphis & $2,087,296$ \\
\hline Asheville & 766,151 & Nashville & $4,600,596$ \\
\hline Boone & 538,170 & Texas (14) & \\
\hline Chapel Hill-Durham & $4,996,743$ & Abilene & 682,570 \\
\hline Charlotte & $2,153,951$ & Austin & $4,219,067$ \\
\hline Fayetteville & 634,126 & Canyon & 899,747 \\
\hline Greensboro & $1,729,099$ & College Station & 812,595 \\
\hline Greenville & $1,023,298$ & Commerce & 605,408 \\
\hline Raleigh & $1,403,434$ & Corpus Christi & 747,629 \\
\hline Winston-Salem & $1,618,747$ & Dallas & $8,239,108$ \\
\hline North Dakota (1) & & El Paso & 956,189 \\
\hline Grand Forks & 509,519 & Galveston & 661,734 \\
\hline Ohio (18) & & Houston & $4,596,864$ \\
\hline Akron & $1,595,648$ & Huntsville & 521,186 \\
\hline Athens & $1,144,112$ & Lubbock & $1,365,848$ \\
\hline Bowling Green & $1,636,219$ & San Antonio & $2,311,777$ \\
\hline Canton & $1,512,423$ & Waco & 997,516 \\
\hline Cincinnati & $5,592,171$ & Utah (3) & \\
\hline Cleveland & $9,142,394$ & Logan & 561,698 \\
\hline Columbus & $7,261,341$ & Provo & $1,095,405$ \\
\hline Dayton & $3,189,778$ & Salt Lake City & $3,773,746$ \\
\hline Delaware & 845,605 & Vermont (2) & \\
\hline Kent & $1,100,213$ & Burlington & $1,037,185$ \\
\hline Lima & 788,486 & $\begin{array}{l}\text { Montpelier } \\
\text { Virginia (5) }\end{array}$ & 918,346 \\
\hline $\begin{array}{l}\text { Oberlin } \\
\text { Oxford }\end{array}$ & $\begin{array}{l}1,411,600 \\
1,067,620\end{array}$ & $\begin{array}{c}\text { Virginia (5) } \\
\text { Arlington }\end{array}$ & $2,916,734$ \\
\hline Springfield & $1,195,714$ & Charlottesville & $2,359,824$ \\
\hline Toledo & $2,460,024$ & Norfolk & $2,762,843$ \\
\hline Wooster & 918,807 & Richmond & $2,777,141$ \\
\hline Youngstown & $1,382,065$ & Roanoke & $1,966,640$ \\
\hline Zanesville & 697,733 & Washington (6) & \\
\hline Oklahoma (3) & & Olympia & $1,069,806$ \\
\hline Oklahoma City & $3,242,373$ & Pullman & 853,458 \\
\hline Stillwater & $1,461,912$ & Seattle & $5,525,875$ \\
\hline Tulsa & $1,508,558$ & Spokane & $1,151,320$ \\
\hline Oregon (5) & & Tacoma & $1,253,235$ \\
\hline Corvallis & 741,003 & Walla Walla & 714,114 \\
\hline Eugene & $1,305,048$ & West Virginia (2) & \\
\hline Medford & 500,723 & Charleston & $1,788,628$ \\
\hline Portland & $2,690,920$ & Morgantown & $1,745,301$ \\
\hline Salem & $1,043,685$ & Wisconsin (5) & \\
\hline Pennsylvania (9) & & Green Bay & 886,991 \\
\hline Bethlehem & $2,276,772$ & Madison & $4,813,740$ \\
\hline Carlisle Barracks & $1,683,676$ & Milwaukee & $5,046,357$ \\
\hline Erie & $1,209,573$ & Oshkosh & $1,355,814$ \\
\hline Harrisburg & $2,195,208$ & Stevens Point & 699,444 \\
\hline Lancaster & $1,526,941$ & Wyoming (1) & \\
\hline Philadelphia & $14,759,903$ & Laramie & 774,593 \\
\hline
\end{tabular}


14 each, and Illinois with 11.

The complete data, presented in Table 1 , indicate the relative rank of the centers in each of the three periods investigated and the number of volumes held by the various centers. Among the first dozen in the top group, changes in relative standing have been minor, with a few exceptions; most notable are the new rankings for the San Jose-Stanford area, Minneapolis, San Francisco, and Pittsburgh. New York, Washington, D.C., and Boston have remained the leading three throughout the nearly four decades.

Below the leaders, numerous shifts in rank may be observed. Dallas has come up from thirty-seventh to fifteenth; San Diego from seventieth to twenty-fifth; Houston from sixty-third to thirty-seventh; Miami from eightyeighth to forty-ninth. Among the first 100 centers, 77 did not possess a suffcient number of volumes to be listed by Wilson in 1935.

At approximately the same date as the Wilson study, another investigation took place to determine the distribution of library resources by states. ${ }^{3}$ More emphasis here was placed on collections of possible research importance. The specifics for inclusion were "all educational libraries with over 25,000 volumes, all public libraries over 50,000 , and all special libraries of a research nature."

Table 3 brings down to 1973 the number of volumes held by the individual states, according to the foregoing criteria, with relative rankings for 1935 and 1955 . Table 4 notes the percentage

TABLE 3

Number of Volumes in U.S. Research Libraries, by States

\begin{tabular}{lcccccr}
\hline \hline \multicolumn{1}{c}{ State } & $\begin{array}{c}\text { Rank } \\
1973\end{array}$ & $\begin{array}{c}\text { Volumes } \\
1973\end{array}$ & $\begin{array}{c}\text { Rank } \\
1955\end{array}$ & $\begin{array}{c}\text { Volumes } \\
1955\end{array}$ & $\begin{array}{c}\text { Rank } \\
1935\end{array}$ & \multicolumn{1}{c}{$\begin{array}{c}\text { Volumes } \\
1935\end{array}$} \\
\hline New York & 1 & $90,431,000$ & 1 & $34,041,000$ & 1 & $16,931,000$ \\
California & 2 & $80,638,000$ & 3 & $23,951,000$ & 2 & $14,401,000$ \\
Massachusetts & 3 & $78,484,000$ & 4 & $22,951,000$ & 3 & $12,944,000$ \\
Ohio & 4 & $42,909,000$ & 5 & $18,606,000$ & 5 & $9,669,000$ \\
Illinois & 5 & $40,023,000$ & 6 & $17,089,000$ & 6 & $8,907,000$ \\
District of Columbia & 6 & $38,773,000$ & 2 & $25,357,000$ & 4 & $10,348,000$ \\
Pennsylvania & 7 & $31,253,000$ & 7 & $14,697,000$ & 7 & $8,056,000$ \\
Texas & 8 & $30,733,000$ & 11 & $6,716,000$ & 16 & $2,196,000$ \\
Michigan & 9 & $24,063,000$ & 8 & $8,988,000$ & 8 & $4,427,000$ \\
New Jersey & 10 & $21,875,000$ & 10 & $8,080,000$ & 10 & $4,137,000$ \\
Indiana & 11 & $17,928,000$ & 12 & $6,520,000$ & 12 & $3,345,000$ \\
Missouri & 12 & $17,879,000$ & 13 & $6,239,000$ & 11 & $3,495,000$ \\
Maryland & 13 & $17,086,000$ & 18 & $3,888,000$ & 17 & $2,163,000$ \\
Connecticut & 14 & $16,545,000$ & 9 & $8,507,000$ & 9 & $4,220,000$ \\
North Carolina & 15 & $15,851,000$ & 19 & $3,882,000$ & 25 & $1,131,000$ \\
Wisconsin & 16 & $14,935,000$ & 14 & $6,195,000$ & 13 & $3,027,000$ \\
Minnesota & 17 & $13,951,000$ & 15 & $5,700,000$ & 14 & $2,916,000$ \\
Virginia & 18 & $13,762,000$ & 20 & $3,684,000$ & 22 & $1,552,000$ \\
Florida & 19 & $13,724,000$ & 26 & $2,553,000$ & 35 & 447,000 \\
Georgia & 20 & $13,616,000$ & 25 & $2,659,000$ & 31 & 790,000 \\
Washington & 21 & $12,615,000$ & 16 & $4,415,000$ & 18 & $1,665,000$ \\
Kentucky & 22 & $11,827,000$ & 29 & $2,379,000$ & 27 & $1,011,000$ \\
Tennessee & 23 & $10,859,000$ & 28 & $2,412,000$ & 20 & $1,642,000$ \\
Louisiana & 24 & $10,246,000$ & 24 & $2,797,000$ & 30 & 818,000 \\
Iowa & 25 & $9,037,000$ & 17 & $4,108,000$ & 15 & $2,527,000$ \\
Colorado & 26 & $9,016,000$ & 21 & $3,098,000$ & 23 & $1,425,000$ \\
Kansas & 27 & $8,229,000$ & 22 & $3,045,000$ & 19 & $1,643,000$ \\
Alabama & 28 & $7,746,000$ & 30 & $2,292,000$ & 32 & 763,000 \\
Oklahoma & 29 & $7,355,000$ & 32 & $1,902,000$ & 33 & 720,000 \\
Oregon & 30 & $6,084,000$ & 23 & $3,038,000$ & 24 & $1,416,000$ \\
Arizona & 31 & $6,014,000$ & 38 & $1,187,000$ & 38 & 425,000 \\
Utah & 32 & $5,656,000$ & 36 & $1,330,000$ & 36 & 446,000
\end{tabular}


TABLE 3-Continued

\begin{tabular}{|c|c|c|c|c|c|c|}
\hline State & $\begin{array}{l}\text { Rank } \\
1973 \\
\end{array}$ & $\begin{array}{c}\text { Volumes } \\
1973\end{array}$ & $\begin{array}{l}\text { Rank } \\
1955 \\
\end{array}$ & $\begin{array}{c}\text { Volumes } \\
1955\end{array}$ & $\begin{array}{c}\text { Rank } \\
1935 \\
\end{array}$ & $\begin{array}{c}\text { Volumes } \\
1935 \\
\end{array}$ \\
\hline West Virginia & 33 & $4,869,000$ & 37 & $1,207,000$ & 39 & 420,000 \\
\hline Rhode Island & 34 & $4,182,000$ & 27 & $2,469,000$ & 21 & $1,640,000$ \\
\hline Mississippi & 35 & $4,176,000$ & 42 & 848,000 & 43 & 267,000 \\
\hline Arkansas & 36 & $4,033,000$ & 39 & $1,153,000$ & 45 & 236,000 \\
\hline South Carolina & 37 & $3,915,000$ & 35 & $1,483,000$ & 34 & 594,000 \\
\hline Maine & 38 & $3,622,000$ & 33 & $1,706,000$ & 26 & $1,046,000$ \\
\hline New Hampshire & 39 & $3,553,000$ & 34 & $1,693,000$ & 28 & 948,000 \\
\hline Nebraska & 40 & $3,311,000$ & 31 & $1,931,000$ & 29 & 947,000 \\
\hline Hawaii & 41 & $3,050,000$ & 40 & 988,000 & 42 & 297,000 \\
\hline Montana & 42 & $2,726,000$ & 42 & 848,000 & 40 & 376,000 \\
\hline $\begin{array}{l}\text { Idaho } \\
\text { a }\end{array}$ & 43 & $2,428,000$ & 49 & 378,000 & 49 & 167,000 \\
\hline New Mexico & 44 & $2,343,000$ & 44 & 677,000 & 50 & 90,000 \\
\hline Vermont & 45 & $2,215,000$ & 41 & 928,000 & 37 & 440,000 \\
\hline Delaware & 46 & $1,861,000$ & 45 & 557,000 & 41 & 314,000 \\
\hline South Dakota & 47 & $1,600,000$ & 46 & 475,000 & 46 & 227,000 \\
\hline North Dakota & 48 & $1,549,000$ & 48 & 456,000 & 44 & 254,000 \\
\hline Wyoming & 49 & $1,221,000$ & 47 & 470,000 & 47 & 187,000 \\
\hline Nevada & 50 & $1,132,000$ & 50 & 249,000 & 48 & 175,000 \\
\hline Alaska & 51 & 766,029 & 51 & 58,000 & & \\
\hline Total & & $791,696,000$ & & $280,860,000$ & & $138,228,000$ \\
\hline
\end{tabular}

TABLE 4

Rate of INCREase of Volumes in Research Libraries OF THE UNITED States FROM 1955 To 1973

\begin{tabular}{lcclccc}
\hline \hline \multicolumn{1}{c}{ State } & $\begin{array}{c}\text { Rank } \\
1973\end{array}$ & $\begin{array}{c}\text { Percent } \\
\text { of Increase }\end{array}$ & \multicolumn{1}{c}{ State } & $\begin{array}{c}\text { Rank } \\
1973\end{array}$ & $\begin{array}{c}\text { Percent } \\
\text { of Increase }\end{array}$ \\
\hline Alaska & 1 & 1320 & Montana & 27 & 322 \\
Idaho & 2 & 644 & Hawaii & 28 & 310 \\
Florida & 3 & 540 & Colorado & 29 & 291 \\
Georgia & 4 & 514 & Missouri & 30 & 287 \\
Arizona & 5 & 507 & Washington & 31 & 286 \\
Mississippi & 6 & 505 & Indiana & 32 & 275 \\
Kentucky & 7 & 498 & New Jersey & 33 & 271 \\
Texas & 8 & 457 & Kansas & 34 & 270 \\
Nevada & 9 & 455 & Michigan & 35 & 268 \\
Tennessee & 10 & 450 & New York & 36 & 266 \\
Maryland & 11 & 438 & South Carolina & 37 & 264 \\
Utah & 12 & 426 & Wyoming & 38 & 260 \\
North Carolina & 13 & 408 & Minnesota & 39 & 245 \\
West Virginia & 14 & 402 & Wisconsin & 40 & 241 \\
Oklahoma & 15 & 387 & Vermont & 41 & 238 \\
Virginia & 16 & 373 & Illinois & 42 & 234 \\
Louisiana & 17 & 367 & Ohio & 43 & 230 \\
Arkansas & 18 & 350 & Pennsylvania & 44 & 224 \\
Rhode Island & 19 & 347 & Iowa & 45 & 220 \\
New Mexico & 20 & 346 & Maine & 46 & 215 \\
Massachusetts & 21 & 342 & New Hampshire & 47 & 210 \\
North Dakota & 22 & 339 & Connecticut & 48 & 205 \\
Alabama & 23 & 338 & Oregon & 49 & 200 \\
South Dakota & 24 & 337 & Nebraska & 50 & 171 \\
California & 25 & 336 & District of Columbia & 51 & 147 \\
Delaware & 26 & 334 & National Average & & 332 \\
\hline
\end{tabular}


of increase for each state between 1955 and 1973. Alaska and Hawaii appear in the tables for the first time, since they had not yet become states when the earlier studies were made.

The extraordinary expansion of holdings in the various states is revealed by the new figures. Except for the District of Columbia, which slipped from second to sixth position, and Connecticut, which dropped from ninth to fourteenth, the top rankings were little changed. Texas made a spectacular jump from eleventh to eighth.

On the basis of holdings by states, according to the criteria described, the na- tion's library resources went from 138,228,000 volumes in 1935 to $280,860,000$ in 1955 to $791,696,000$ in 1973 , nearly tripling in the past two decades. In percentage of increase, the southern, southwestern, and northwestern states were in the lead. None of the states except Alaska held less than one million volumes.

Another approach to the matter of distribution of resources is holdings by geographic regions of the country. The number of volumes in each of the six principal regions of the United States in 1955 and in 1973 is recorded in Table 5 . Though the Northeast held 39

TABLE 5

Number of Volumes in Research Libraries of the Untted States BY Geographic Area IN 1955 AND IN 1973

\begin{tabular}{|c|c|c|c|c|c|}
\hline & & mes & & & \\
\hline & 1973 & 1955 & & 1973 & 1955 \\
\hline \begin{tabular}{l} 
Northeast \\
Connecticut \\
Delaware \\
District of Columbia \\
Maine \\
Maryland \\
Massachusetts \\
New Hampshire \\
New Jersey \\
New York \\
Pennsylvania \\
Rhode Island \\
Vermont \\
West Virginia \\
\multicolumn{1}{c}{ Total }
\end{tabular} & $\begin{array}{r}16,545,000 \\
1,861,000 \\
38,773,000 \\
3,622,000 \\
17,086,000 \\
78,484,000 \\
3,553,000 \\
21,875,000 \\
90,431,000 \\
31,253,000 \\
4,182,000 \\
2,215,000 \\
4,869,000 \\
314,749,000\end{array}$ & $\begin{array}{r}8,507,000 \\
557,000 \\
25,357,000 \\
1,706,000 \\
3,888,000 \\
22,951,000 \\
1,693,000 \\
8,080,000 \\
34,041,000 \\
14,697,000 \\
2,469,000 \\
928,000 \\
1,207,000 \\
126,081,000\end{array}$ & \begin{tabular}{l} 
Southeast \\
Alabama \\
Arkansas \\
Florida \\
Georgia \\
Kentucky \\
Louisiana \\
Mississippi \\
North Carolina \\
South Carolina \\
Tennessee \\
Virginia \\
\multicolumn{1}{c}{ Total }
\end{tabular} & $\begin{array}{r}7,746,000 \\
4,033,000 \\
13,724,000 \\
13,616,000 \\
11,827,000 \\
10,246,000 \\
4,176,000 \\
15,851,000 \\
3,915,000 \\
10,859,000 \\
13,762,000 \\
109,755,000\end{array}$ & $\begin{array}{r}2,292,000 \\
1,153,000 \\
2,553,000 \\
2,659,000 \\
2,379,000 \\
2,797,000 \\
828,000 \\
3,882,000 \\
1,483,000 \\
2,412,000 \\
3,684,000 \\
26,122,000\end{array}$ \\
\hline $\begin{array}{l}\text { Midwest } \\
\text { Illinois } \\
\text { Indiana } \\
\text { Iowa } \\
\text { Michigan } \\
\text { Minnesota }\end{array}$ & $\begin{array}{r}40,023,000 \\
17,928,000 \\
9,037,000 \\
24,063,000 \\
13,951,000\end{array}$ & $\begin{array}{r}17,089,000 \\
6,520,000 \\
4,108,000 \\
8,988,000 \\
5,700,000\end{array}$ & $\begin{array}{l}\text { Northwest } \\
\text { Colorado } \\
\text { Idaho } \\
\text { Kansas } \\
\text { Montana } \\
\text { Nebraska }\end{array}$ & $\begin{array}{l}9,016,000 \\
2,428,000 \\
8,229,000 \\
2,726,000 \\
3,311,000\end{array}$ & $\begin{array}{r}3,098,000 \\
378,000 \\
3,045,000 \\
848,000 \\
1,931,000\end{array}$ \\
\hline $\begin{array}{l}\text { Midwest } \\
\text { Missouri } \\
\text { Ohio } \\
\text { Wisconsin } \\
\text { Total }\end{array}$ & $\begin{array}{r}17,879,000 \\
42,909,000 \\
14,935,000 \\
180,725,000\end{array}$ & $\begin{array}{r}6,239,000 \\
18,606,000 \\
6,195,000 \\
73,445,000\end{array}$ & \begin{tabular}{l} 
Northwest \\
North Dakota \\
South Dakota \\
Utah \\
Wyoming \\
\multicolumn{1}{c}{ Total }
\end{tabular} & $\begin{array}{r}1,549,000 \\
1,600,000 \\
5,656,000 \\
1,221,000 \\
35,736,000\end{array}$ & $\begin{array}{r}456,000 \\
475,000 \\
1,330,000 \\
470,000 \\
12,031,000\end{array}$ \\
\hline \begin{tabular}{l} 
Southwest \\
Arizona \\
New Mexico \\
Oklahoma \\
Texas \\
\multicolumn{1}{c}{ Total }
\end{tabular} & $\begin{array}{r}6,014,000 \\
2,343,000 \\
7,355,000 \\
30,733,000 \\
46,445,000\end{array}$ & $\begin{array}{r}1,187,000 \\
677,000 \\
1,902,000 \\
6,716,000 \\
10,482,000\end{array}$ & \begin{tabular}{l} 
Far West \\
California \\
Nevada \\
Oregon \\
Washington \\
\multicolumn{1}{c}{$\quad$ Total }
\end{tabular} & $\begin{array}{r}80,638,000 \\
1,132,000 \\
6,084,000 \\
12,615,000 \\
100,469,000\end{array}$ & $\begin{array}{r}23,951,000 \\
249,000 \\
3,038,000 \\
4,415,000 \\
31,652,000\end{array}$ \\
\hline
\end{tabular}


percent of the nation's total library resources in 1973, its overwhelming lead, which Wilson noted some thirty-five years ago, has declined in terms of the other regions. Of particular note is the rapid rise of the Southeast, Southwest, and Far West.

The reasons for what may rightly be described as an explosion of library collections in all the American states are doubtless complex. Among the factors that may be cited are the establishment of hundreds of new institutions of higher education, millions of additional students in colleges and universities across the land, increased book budgets in all types of libraries, federal aid to libraries, extensive new foreign acquisition programs, and, finally, a steadily expanding rate of publication of books and journals, to which libraries have responded by stepped-up acquisition activities.

\section{REFERENCES}

1. Louis R. Wilson, Geography of Reading (Chicago: Univ. of Chicago Pr., 1938), p.118-23.

2. College \& Research Libraries, 18:183-89, 235-37 (May 1957).

3. Tommie Dora Barker, Libraries of the South (Chicago: American Library Association, 1936), p.118-19. 